\title{
Understanding and Developing Role-Based Administrative Models
}

\author{
Jason Crampton \\ Information Security Group, Royal Holloway, University of London, England
}

\begin{abstract}
Access control data structures generally need to evolve over time in order to reflect changes to security policy and personnel. An administrative model defines the rules that control the state changes to an access control model and the data structures that model defines. We present a powerful framework for describing role-based administrative models. It is based on the concept of administrative domains and criteria that control state changes in order to preserve certain features of those domains. We define a number of different sets of criteria, each of which control the effect of state changes on the set of administrative domains and thereby lead to different role-based administrative models. Using this framework we are able to identify some unexpected connections between the ARBAC97 and RHA administrative models and to compare their respective properties. In doing so we are able to suggest some improvements to both models.
\end{abstract}

\section{Categories and Subject Descriptors}

D.4.6 [Operating Systems]: Security and Protection-Access controls; H.2.7 [Database Management]: Database Administration-Security, integrity and protection; K.6.5 [Management of Computing and Information Systems]: Security and Protection

\section{General Terms}

Security, Theory

\section{Keywords}

role-based access control, role-based administration, administrative scope, administrative domain, RHA, ARBAC97

\section{INTRODUCTION}

An access control mechanism is a component of a computer system that is used to limit the access that authenticated and authorized users have to the resources provided by that system. An access control model typically defines a

Permission to make digital or hard copies of all or part of this work for personal or classroom use is granted without fee provided that copies are not made or distributed for profit or commercial advantage and that copies bear this notice and the full citation on the first page. To copy otherwise, to republish, to post on servers or to redistribute to lists, requires prior specific permission and/or a fee.

CCS'05, November 7-11, 2005, Alexandria, Virginia, USA.

Copyright 2005 ACM 1-59593-226-7/05/0011 ...\$5.00. collection of sets, functions and relations that represent elements of an access control mechanism. We will refer to such sets as components of the access control model. The components of the Harrison-Ruzzo-Ullman (HRU) model [10], for example, are the set of objects $O$, the set of subjects $S$, the set of access rights $A$, and the protection matrix $M: S \times O \rightarrow 2^{A}$.

A component can be static or dynamic: a static component is one that doesn't change over time, such as the set of access rights $A$ in the protection matrix model; conversely, a dynamic component, such as the set of subjects $S$ in the protection matrix model, does change over time. The state of a model can be thought of as a tuple $\left(C_{1}, \ldots, C_{n}\right)$, where $C_{j}$ is a dynamic component in the model. In the HRU model, for example, the state is defined to be the tuple $(S, O, M)$; the set of access rights is static.

An administrative model for an access control mechanism defines a decision process that determines whether a request to change the state is permitted. Typically, an administrative model is defined by a fixed set of commands, each command containing a conditional statement and a body that is executed if the conditional statement evaluates to true. The body will comprise a number of atomic operations each of which changes a dynamic component of the associated access control model. In the HRU model, for example, the conditional statement checks for the presence of access rights in the matrix and the atomic operations make changes to the rows, columns or entries of the matrix.

Inspired by early work at MITRE Corporation [1] and NIST [8], role-based access control (RBAC) has been the subject of considerable research in recent years resulting in several important models, including the RBAC96 model [16], the role graph model [13], and the NIST model [9]. Many of these ideas were recently consolidated to form the basis for the ANSI RBAC standard [2]. A number of commercial products, such as Trusted Solaris, Windows Authorization Manager, Oracle 9 and Sybase Adaptive Server, implement RBAC.

Despite the enthusiasm for RBAC, the use of RBAC principles to manage RBAC systems has been less widely studied. The models cited above, for example, rely on centralized procedures to change dynamic components of the respective models. The most mature decentralized role-based administrative models are ARBAC97 [15] and the RHA (role hierarchy administration) model [6]. Both models are designed to inter-operate with RBAC96, but could also be applied to ANSI RBAC systems, and both models exploit the structure of the role hierarchy to control changes. Nevertheless, the motivation for the development and design of these models 
has been somewhat vague: the creators of ARBAC97, for example, talk of the need to prevent "anomalous side effects" arising from unconstrained changes to the hierarchy; similarly, Crampton and Loizou state that RHA prevents "unexpected side effects due to inheritance elsewhere in the hierarchy", although this notion is formalized to some extent.

In this paper, we undertake a rigorous analysis of the properties that define the behaviour of role-based administrative models. The analysis is based on the notion of an administrative domain, a self-contained sub-hierarchy of the role hierarchy. Our first major result is to prove that administrative domains are pairwise nested or disjoint. We then define what it means for administrative domains to be preserved. This enables us to define a number of sets of criteria that impose constraints on the functionality of a role-based administrative model. Each of these sets defines a mode of operation for a role-based administrative model, enabling systems and application developers to choose the administrative model best suited to their requirements. Each set of criteria requires that administrative operations must preserve certain structural properties of administrative domains. We might insist, for example, that an administrative role can make changes to its own administrative domain and any domains contained within it. These criteria enable us to classify role-based administrative models according to their permissiveness, and to create a framework for developing role-based administrative models.

One of the most striking consequences of our analysis is to reveal a fundamental and hitherto unexpected connection between RHA and ARBAC97. In particular, we find that RHA is the most permissive of administrative models, whereas ARBAC97 is among the most restrictive. Informally, our analysis enables us to draw a road map from RHA to ARBAC97, identifying interesting features (new models) along the way. We also establish that ARBAC97 is more restrictive than is necessary and point out a number of weaknesses in the original formulation.

In the next section we briefly review the RBAC96 model and some relevant mathematics. We also specify the operational semantics of the operations used in a role-based administrative model. In Section 3 we define administrative scope, the central concept in RHA, and show how this immediately leads to the concept of an administrative domain. We prove that administrative domains must be either disjoint or nested and introduce the idea of a domain tree. In Section 4 we formally define what it means for an administrative domain to be preserved by an administrative operation and introduce the idea of local, hierarchical and universal domain preservation. We also define what it means for an operation to be autonomy preserving. We then introduce three different sets of criteria and state a number of important results in relation to the preservation of administrative domains. In Section 5 we introduce the idea of an administrative role and define a template for constructing role-based administrative models. In this section we note the connection between this template and RHA. In Section 6 we describe the connection between our framework and ARBAC97, and provide a concise characterization of ARBAC97. We also identify a number of flaws in ARBAC97 and describe appropriate remedies. We conclude with an appraisal of our framework and describe the numerous opportunities for further research in this area.

\section{PRELIMINARIES}

\subsection{RBAC96}

RBAC96 is a family of access control models that assumes the existence of a set of roles $R$, a set of permissions $P$, a set of users $U$, and two relations $U A \subseteq U \times R$ and $P A \subseteq P \times R$ that bind users and permissions to roles [16]. These sets and relations form the basis for $\mathrm{RBAC}_{0}$, the simplest model in the RBAC96 family. A request by a user $u$ to invoke permission $p$ is granted if there exists a role $r$ such that $(u, r) \in U A$ and $(p, r) \in P A$.

$\mathrm{RBAC}_{1}$ introduces the concept of a role hierarchy, which is modelled as a partial order on the set of roles. The role hierarchy permits a role $r$ to inherit the permissions assigned to any more junior role. This significantly reduces the administrative burden by reducing the number of explicit assignments that need to be stored in the $U A$ and $P A$ relations. A request by a user to invoke permission $p$ is granted if there exist roles $r$ and $r^{\prime}$ such that $(u, r) \in U A,\left(p, r^{\prime}\right) \in P A$ and $r \geqslant r^{\prime} .\left(\mathrm{RBAC}_{0}\right.$ and $\mathrm{RBAC}_{1}$ have recently been superseded by the core and hierarchical components of the ANSI RBAC standard [2].)

\subsection{Partial orders}

Let $\langle X, \leqslant\rangle$ be a partially ordered set and let $x, y \in X$. We write $x<y$ if $x \leqslant y$ and $x \neq y$. We may write $y \geqslant x$ whenever $x \leqslant y$. We write $x \| y$ if $x \not y$ and $y \nless x$. We say $Y$ is an antichain if for all $y, z \in Z, y \neq z$ implies that $y \| z$. We write $[x, y]$ to denote the range $\{z \in X: x \leqslant z \leqslant y\}$.

We say $y$ covers $x$, or $x$ is covered by $y$, denoted $x \lessdot y$, if $x<y$ and for all $z \in X, x \leqslant z<y$ implies $x=z$. In other words, $x \lessdot y$ is shorthand for " $y$ is an immediate parent of $x$ ". The Hasse diagram of $X$ is the directed graph of the covering relation $(X, \lessdot)$ : in other words, transitive relationships in the poset are implied by paths in the Hasse diagram. In the context of RBAC, the Hasse diagram represents the role hierarchy.

We define $\Delta x=\{y \in X: y \lessdot x\}$ and $\nabla x=\{y \in X: x \lessdot y\}$. In other words, $\Delta x$ is the set of immediate children of $x$ and $\nabla x$ is the set of immediate parents of $x$. It is easy to show that $\Delta x$ and $\nabla x$ are antichains for all $x \in X$. We define $\downarrow x=\{y \in X: y \leqslant x\}$ and $\uparrow x=\{y \in X: x \leqslant y\}$. For $Y \subseteq X$, we define

$$
\downarrow Y=\bigcup_{y \in Y} \downarrow y \quad \text { and } \quad \uparrow Y=\bigcup_{y \in Y} \uparrow y .
$$

The interested reader is referred to the book by Davey and Priestley for an introduction to lattice and order theory [7].

In the context of RBAC, $\downarrow r$ represents the set of roles available to a user assigned to $r$ and $\uparrow r$ represents the set of roles to which the permission $p$ is available if $p$ is assigned to $r$. The expression $\downarrow r \cup \uparrow r$ will be used extensively once we introduce the concept of administrative scope, and will be abbreviated to $\uparrow r$.

\subsection{Administrative operations}

Role-based access control models typically include a role hierarchy, which is modelled as a partial order on the set of roles. The role hierarchy is represented as the set of directed edges in the Hasse diagram of $R$ (an example is shown in Figure 1). Hence, there are two dynamic components: $R$ and the covering relation on $R$. This gives rise to the four 
hierarchy operations: ${ }^{1}$

- addEdge $(a, c, p)$, which adds the directed edge $(c, p)$ to the hierarchy, where $c, p \in R$;

- deleteEdge $(a, c, p)$, which deletes the directed edge $(c, p)$ from the hierarchy;

- addRole $(a, r, C, P)$, which creates the role $r$ with immediate children $C \subseteq R$ and immediate parents $P \subseteq R$;

- deleteRole $(a, r)$, which deletes the role $r \in R$.

In addition we have the following assignment operations, each of which is assumed to be performed by administrative role $a$.

- $\operatorname{addUA}(a, u, r)$, which adds the pair $(u, r)$ to the $U A$ relation;

- deleteUA $(a, u, r)$, which deletes the pair $(u, r)$ from the $U A$ relation;

- $\operatorname{addPA}(a, p, r)$, which adds the pair $(p, r)$ to the $P A$ relation;

- deletePA $(a, p, r)$, which deletes the pair $(p, r)$ from the $P A$ relation.

Collectively we refer to these eight operations as administrative operations. In this paper, we will focus on the hierarchy operations; experience has shown that it is straightforward to incorporate the other operations [6].

Informally, the execution of a hierarchy operation will affect one or more roles in the hierarchy. The set of roles that are affected by an operation is not necessarily immediately obvious, because of the transitivity implied by the role hierarchy. It may be necessary, for example, to "repair" the hierarchy relation following addEdge and deleteEdge operations in order to remove redundancy and to preserve inheritance, respectively. Table 1 summarizes the changes to $R$ and $R H$ caused by hierarchy operations. The approach described in this table assumes that only the covering relation is stored. ${ }^{2}$

\section{ADMINISTRATIVE SCOPE}

The RBAC96 model does not provide any model for controlling updates to the role hierarchy and the assignment relations. This omission was addressed by the ARBAC97 model [15], which provides a role-based model for administering a role-based access control system. However, the ARBAC97 model suffers from its inability to manage many types of hierarchies [6, Section 8]. Crampton and Loizou introduced the RHA model ${ }^{3}$ as a more flexible and widely applicable alternative to ARBAC97 [6].

\footnotetext{
${ }^{1}$ In $\mathrm{RBAC}_{0}$, the only dynamic component is the set of roles (since the set is unordered), so we only require two operations: one to add a role and one to delete a role.

${ }^{2}$ Of course, the full order relation could be stored, which would make the deletion of an edge quite straightforward. However, this approach would require several edges to be added to the relation when a new edge or role is added, in order to preserve transitivity. In short, there are a number of ways of representing the role hierarchy, each of which influences the complexity of different hierarchy operations in different ways.

${ }^{3}$ In fact there are four different RHA models of differing complexity. For convenience we will refer to the RHA model, except in Section 5, when we discuss the particular members of the family.
}

The RHA model is based around the idea of administrative scope. Every role $r \in R$ has an administrative scope, which defines the set of roles that can be modified by $r$. Administrative scope is determined by the structure of the hierarchy. Informally, $r^{\prime}$ is in the administrative scope of $r$ if any change to $r^{\prime}$ will only be observed by $r$ and roles more senior than $r$. That is, any change to $r^{\prime}$ made by $r$ will not have unexpected side effects due to inheritance elsewhere in the hierarchy. More formally, we have the following definition [6].

DeFinition 1. The administrative scope of a role $r$, denoted $\sigma(r)$, is defined to be

$$
\sigma(r)=\{s \in \downarrow r: \uparrow s \subseteq \uparrow r\} .
$$

The strict administrative scope of $r$ is defined to be $\sigma(r) \backslash\{r\}$ and is denoted $\widehat{\sigma}(r)$. For $A \subseteq R$ we define $\sigma(A)=\{r \in \downarrow A: \uparrow r \subseteq \uparrow A\}$ and $\widehat{\sigma}(A)=\sigma(A) \backslash A$.

Note that $r \in \sigma(r)$ for all $r$, which motivates the definition of strict administrative scope. In the role hierarchy depicted in Figure 1, for example, $\sigma(\mathrm{PL} 1)=\{$ ENG1, PE1, QE1, PL1 $\}$.

\subsection{Administrative scope and administrative operations}

The conditions that determine whether an administrative operation is allowed to proceed in the RHA model are summarized in Table 2. Note that each condition requires that the arguments of the operation be contained in the administration scope of the role performing the operation.

\subsection{Administrative domains}

We will say $D \subseteq R$ is an administrative domain, with administrator $r$, if $D=\sigma(r)$ for some $r \in R$. We will write $\mathcal{D}_{R}$ for the set of administrative domains in $R$. Henceforth we will omit $R$ when it is obvious from context.

In this section we establish a fundamental result concerning administrative domains: namely, that each pair of domains is either nested or disjoint. This leads naturally to the concept of an administrative domain tree and of the smallest domain containing a given role. These concepts will be used extensively in the following section.

Lemma 2. Let $a, b \in R$. Then

$$
\sigma(a) \cap \sigma(b)= \begin{cases}\sigma(a) & \text { if } a \in \sigma(b), \\ \sigma(b) & \text { if } b \in \sigma(a), \\ \emptyset & \text { otherwise. }\end{cases}
$$

Proof. Let $r \in \sigma(a)$. We consider each of the three cases in turn. (Note that $\sigma(a) \cap \sigma(b)=\sigma(a)$ is equivalent to saying that $\sigma(a) \subseteq \sigma(b)$.)

If $a \in \sigma(b)$ then, by definition, $a \leqslant b, \downarrow a \subseteq \downarrow b$ and $\uparrow a \subseteq \uparrow b$. Hence $\uparrow r \subseteq \uparrow a \subseteq \uparrow b$ and $r \in \sigma(b)$. By symmetry, $\sigma(a) \cap \sigma(b)=\sigma(\bar{b})$ if $b \in \sigma(a)$.

Now assume that $a \notin \sigma(b)$ and $b \notin \sigma(a)$. Note that $a \neq b$. If $a \| b$, then $a \notin \uparrow b$; by definition, $r \in \sigma(a)$ implies $r \leqslant a$ (that is, $a \in \uparrow r$ ) and hence $r \notin \sigma(b)$. Otherwise, we can assume without loss of generality that $a<b$. Then since $a \notin \sigma(b)$, there exists $x \in \uparrow a$ such that $x \notin \uparrow b$. Now given that $r \in \sigma(a)$, we have $r \leqslant a$, and hence $r \leqslant x$ by transitivity. Therefore $x \in \uparrow r$ and hence $r \notin \sigma(b)$.

REMARK 3. Note that $a \leqslant b$ does not imply that $\sigma(a) \subseteq \sigma(b)$. A counterexample is provided by ED and PL1 in Figure 1. 


\begin{tabular}{|c|c|}
\hline Operation & Semantics \\
\hline $\operatorname{addEdge}(a, c, p)$ & $\begin{array}{l}R H \leftarrow R H \cup\{(c, p)\} \backslash\{(x, p): x \in \Delta c \cap \Delta p\} \backslash\{(c, y): y \in \nabla c \cap \nabla p\} \\
\text { If }(r, c),(r, p) \in R H \text { and the edge }(c, p) \text { is added, then we no longer require the edge }(r, p) \text {, } \\
\text { because }(r, c) \text { and }(c, p) \text { imply }(r, p) \text { by transitivity. Hence any role } r \text { that is an immediate } \\
\text { child of both } c \text { and } p \text { is affected by the operation as the edge }(r, p) \text { must be deleted from } R H \text {. } \\
\text { Similarly, any role } r \text { that is an immediate parent of both } c \text { and } p \text { is affected by addEdge }(a, c, p) \\
\text { as the edge }(c, r) \text { must be deleted from } R H \text {. }\end{array}$ \\
\hline \multirow[b]{2}{*}{ deleteEdge $(a, c, p)$} & $R H \leftarrow R H \backslash\{(c, p)\} \cup\{(x, p): x \in \Delta c\} \cup\{(c, y): y \in \nabla p\}$ \\
\hline & $\begin{array}{l}\text { If }(r, c) \in R H \text {, then } r \lessdot c \lessdot p \text { and hence we may need to add the edge }(r, p) \text { to preserve the } \\
\text { inheritance. Similarly, if }(p, r) \in R H \text {, then } c \lessdot p \lessdot r \text { and we may need to add the edge }(c, r) \text {. }\end{array}$ \\
\hline \multirow[b]{2}{*}{$\operatorname{addRole}(a, r, C, P)$} & $\begin{array}{l}R \leftarrow R \cup\{r\} \\
R H \leftarrow R H \cup\{(c, r): c \in C\} \cup\{(r, p): p \in P\} \backslash\{(c, p): c \in C, p \in P\} \\
\end{array}$ \\
\hline & $\begin{array}{l}\text { If }(c, p) \in R H, \text { where } c \in C \text { and } p \in P \text {, then the edge }(c, p) \text { becomes redundant following the } \\
\text { addition of role } r \text { (since }(c, r) \text { and }(r, p) \text { are added to the hierarchy, thereby implying } c<p \text { by } \\
\text { transitivity). Hence we remove }(c, p) \text { from } R H \text {. }\end{array}$ \\
\hline \multirow{3}{*}{ deleteRole $(a, r)$} & $R \leftarrow R \backslash\{r\}$ \\
\hline & $R H \leftarrow R H \cup\{(c, p): c \in \Delta r, p \in \nabla r\}$ \\
\hline & $\begin{array}{l}\text { For any role } c \in \Delta r \text { and any role } p \in \nabla r \text { we have } c \lessdot r \lessdot p \text {, so we must add an edge }(c, p) \text { to } \\
\text { the hierarchy following the deletion of } r \text {. }\end{array}$ \\
\hline
\end{tabular}

Table 1: Operational semantics of hierarchy operations

\begin{tabular}{|l|l|}
\hline Operation & Conditions \\
\hline addRole $(a, r, C, P)$ & $C \subseteq \widehat{\sigma}(a), P \subseteq \sigma(a)$ \\
deleteRole $(a, r)$ & $r \in \widehat{\sigma}(a)$ \\
\hline addEdge $(a, c, p)$ & $c, p \in \sigma(a)$ \\
deleteEdge $(a, c, p)$ & $c, p \in \sigma(a)$ \\
\hline
\end{tabular}

Table 2: Conditions for success of hierarchy operations in RHA

Lemma 2 states that administrative domains are either nested or disjoint. ${ }^{4}$ An illustration of this result is given in Figure 1(b); domains are enclosed by broken lines. The hierarchy depicted in Figure 1(a) is adapted from an example by Sandhu. Hence, for any partially ordered set of roles $R$, the partially ordered set $\langle\mathcal{D}, \subseteq\rangle$ is a tree. Figure $1(\mathrm{c})$ illustrates the administrative domain tree for the role hierarchy depicted in Figure 1.

The administrative domain tree provides a natural ordering on the set of administrators. Specifically, if $a$ and $b$ are administrators, we write $a \preccurlyeq b$ if $\sigma(a) \subseteq \sigma(b)$. Figure $1(\mathrm{~d})$ shows the partial order defined on the set of administrators.

Note that $\{r\}$ is an administrative domain (with administrator $r$ ) for any $r$ that is non-maximal in another administrative domain (as is the case with PE1, for example). We call such administrative domains trivial. Note also that, by the conditions in Table 2, the administrator of a trivial administrative domain cannot perform any hierarchy operation. Henceforth, we confine our attention to non-trivial administrative domains.

\footnotetext{
${ }^{4}$ ARBAC97 introduces the concept of authority ranges which are defined by the administrator of the system. It is required that authority ranges are either nested or disjoint. It is interesting that this property "comes for free" with domains defined using administrative scope. We will consider this in more detail in Section 6.
}

\section{PRESERVING ADMINISTRATIVE SCOPE}

In Section 2.3 we observed that the effect of a hierarchy operation is not necessarily limited to the parameters of the operation. A consequence of this is that the administrative scope of a role can change following a hierarchy operation. If, for example, PL1 deletes the edge (PE1, PL1), a new edge (PE1, DIR) is added to preserve inheritance and PE1 no longer belongs to $\sigma$ (PL1).

Of course, these operational semantics may be regarded as acceptable in certain situations. However, if we assume that it is desirable for a hierarchy operation to preserve administrative scope, then it is necessary to impose some additional conditions that must be satisfied if the operation is to succeed. There are at least three different possibilities. Specifically, if a performs a hierarchy operation we could require that:

- $\sigma(a)$ should be preserved;

- $\sigma\left(a^{\prime}\right)$ should be preserved for all $a^{\prime} \geqslant a$;

- $\sigma\left(a^{\prime}\right)$ should be preserved for all $a^{\prime}$.

\subsection{Scope preserving hierarchy operations}

A hierarchy operation may cause a change to $R$ or the partial ordering defined on $R$. If $S \subseteq R$, we will write $S^{\prime}$ or $(S)^{\prime}$ to denote the value of $S$ following a hierarchy operation. In particular, we will write $\sigma(a)^{\prime}$ to denote the administrative scope of $a$ following an operation, but for clarity we 


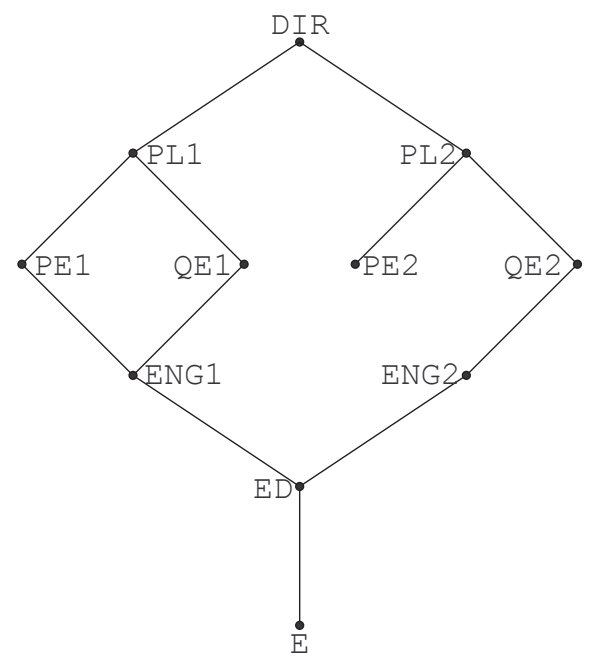

(a) Role names

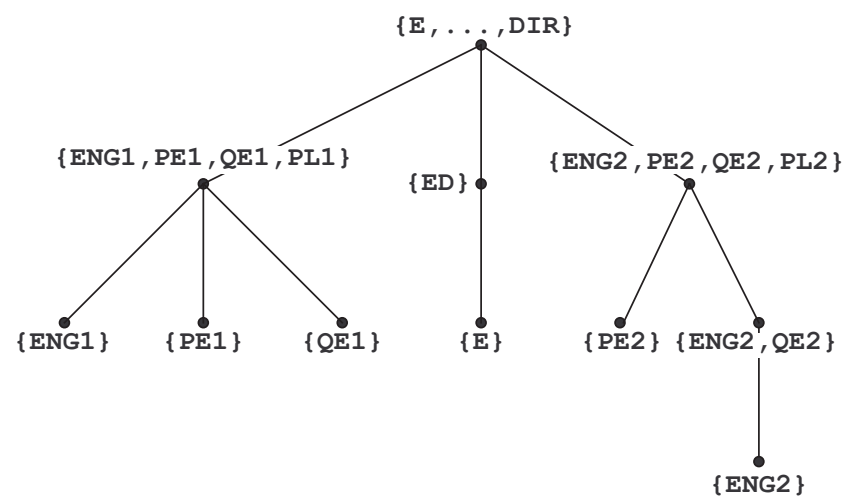

(c) Administrative domain tree

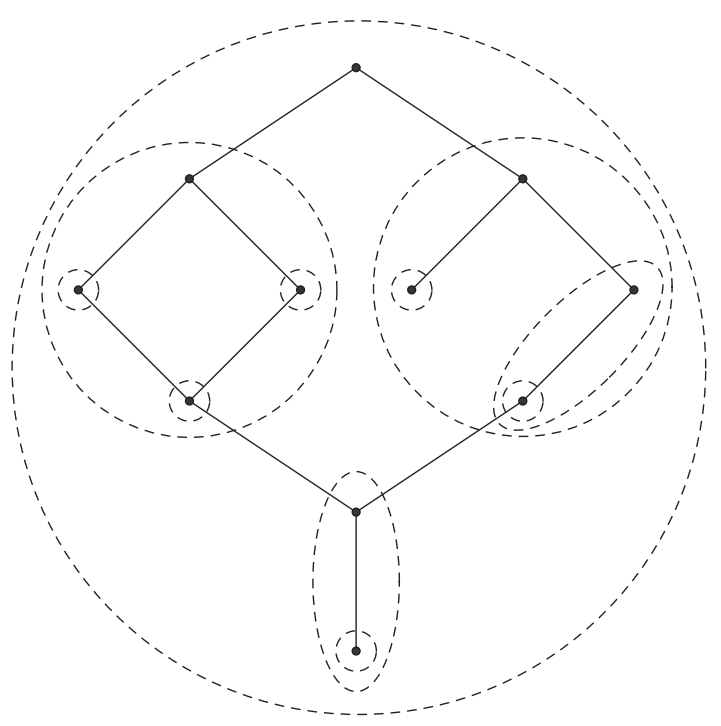

(b) Administrative domains

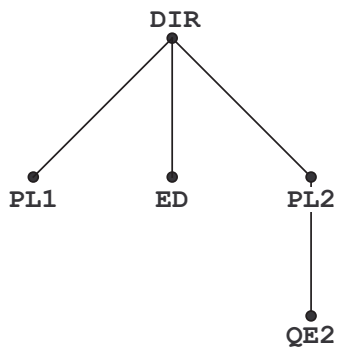

(d) Administrator tree

\section{Figure 1: An example role hierarchy}

prefer to write $(\uparrow x)^{\prime},(\downarrow x)^{\prime}$ and $(\uparrow x)^{\prime}$ (rather than $\uparrow x^{\prime}, \downarrow x^{\prime}$ and $\left.\uparrow x^{\prime}\right)$. Informally, we say $S$ is preserved by a hierarchy operation if anything in $S$ prior to the operation remains in $S$ if it remains in $R$. More formally, we have the following definition.

Definition 4. Let $S \subseteq R$. We say $S$ is preserved by a hierarchy operation if $S \cap R^{\prime} \subseteq S^{\prime}$.

DEFINITION 5. We say an operation performed by a is

- locally scope preserving if it preserves $\sigma(a)$;

- hierarchically scope preserving if it preserves $\sigma(b)$ for all $b \in R$ such that $\sigma(a) \subseteq \sigma(b)$;

- universally scope preserving if it preserves $\sigma(b)$ for all $b \in R$.
For convenience, we will say an operation is 0SP if it is always locally scope preserving, 1SP if it is always hierarchically scope preserving, and 2SP if it is always universally scope preserving. It is clear from the definition that if an operation is 2SP then it is also OSP and 1SP, and that if an operation is 1SP then it is also 0SP.

Note that hierarchy operations are not, in general, OSP. The operation deleteEdge(PL1, PE1, PL1) defined in Table 2 is not 0SP, since $\sigma$ PL1) $=\{$ ENG1, QE1, PE1, PL1 $\}$ and $\sigma(\mathrm{PL} 1)^{\prime}=\{\mathrm{QE} 1, \mathrm{PL} 1\}$. Hence it is necessary to impose restrictions on the hierarchy operations that are permitted to succeed (if we wish to preserve administrative scope). We address these issues in the next section and also specify conditions that define 0SP, 1SP and 2SP operations. 
DEFINITION 6. We say an operation performed by a is autonomy preserving if there does not exist $b \leqslant a$ such that $b$ is permitted to perform the same operation.

We say an operation is 3SP if it is autonomy preserving. An example will make this notion clear: let $a$ and $b$ be administrators with $\sigma(b) \subseteq \sigma(a)$ and $r \in \sigma(b)$; then deleteRole $(a, r)$ succeeds if the operation is 2SP but fails if it is 3SP. In other words, a 3SP operation will only succeed if it is invoked by the most local administrator: senior administrators cannot change nested administrative domains within their scope.

\subsection{Scope preserving administrative models}

An administrative model $\mathcal{M}$ is part of the reference monitor that determines whether requests to perform administrative operations should succeed. Typically, $\mathcal{M}$ specifies conditions for each hierarchy operation that must be satisfied for that operation to succeed (as in Table 2, for example). We say a hierarchy operation is $\mathcal{M}$-permissible if the condition(s) permit the operation to proceed. Some conditions may only preserve the administrative scope of the role that performs the operation, while others may preserve the administrative scope of all roles. We now introduce a classification scheme for administrative models by extending the definitions of OSP, 1SP, 2SP and 3SP for hierarchy operations in the natural way.

Definition 7. We say that $\mathcal{M}$ is iSP if all $\mathcal{M}$ permissible hierarchy operations are $i \mathrm{SP}, 0 \leqslant i \leqslant 3$.

The RHA family of models is not OSP. This is a potential criticism of the RHA family of models, although it should be noted that a role can never increase its own administrative scope by performing a hierarchy operation. Nevertheless, we believe this provides sufficient motivation for introducing the idea of OSP.

Informally, we note that one problem with the set of conditions in Table 2 is that deleting an edge can "break" the administrative scope of the role performing the deletion. This problem arises because the operation affects roles outside the administrative scope of the role performing the deletion. In the case of the operation deleteEdge(PL1, PE1, PL1), the operation affects DIR which is not in $\sigma$ (PL1).

However, a OSP model does not necessarily prevent a role $a$ from performing a hierarchy operation that preserves $\sigma(a)$ but does not preserve the administrative scope of a more senior role. In many situations, we would not want this to happen, hence the idea of 1SP models.

Note that a 1SP model would permit the operation addRole(DIR, $\{\mathrm{QE} 1\},\{\mathrm{DIR}\})$, which does not preserve $\sigma$ (PL1). As a further example, deleteEdge(DIR, ENG1, QE1) is 2SP, but deleteEdge(DIR, QE1, PL1) is not. There may be situations when we wish to guarantee the autonomy of administrative domains, for example - where we want the administrative scope of every role to be preserved by every hierarchy operation; hence the introduction of 2SP models. (We shall see later that ARBAC97 is approximately 2SP, although it was never characterized in this way when it was introduced.)

Finally, we note that a 2SP model would permit the operation deleteRole(DIR, QE1). Although this operation preserves $\sigma$ (PL1), since $\sigma$ (PL1) $\cap R^{\prime}=\sigma\left(\right.$ PL1) ${ }^{\prime}$, we may wish to strengthen the autonomy of domains by preventing more senior administrators changing nested domains and hence we introduce the idea of 3SP models.

\subsection{Scope preserving conditions}

In this section we provide several different sets of conditions governing the success of hierarchy operations. We first introduce a number of useful consequences of Lemma 2. We then state without proof a number of results concerning the scope preserving properties of each of these sets of conditions.

A corollary of Lemma 2 is that for every role $r \in R$, there exists a smallest (non-trivial) administrative domain to which $r$ belongs, which we will denote by $[r] .^{5}$ Since $[r]$ is an administrative domain, $[r]=\sigma(a)$ for some role $a$, and we will say that $a$ is the line manager of role $r$. From Figure $1(\mathrm{c})$ we see that $[\mathrm{PE} 1]=\{\mathrm{ENG} 1, \mathrm{PE} 1, \mathrm{QE} 1, \mathrm{PL} 1\}$, for example, and hence that PL1 is the line manager of PE1.

Let $X \subseteq R$. We define $\lfloor X\rfloor$ to be the largest administrative domain $D$ such that $D \subseteq[x]$ for all $x \in X$, and $\lceil X\rceil$ to be the smallest administrative domain $D$ such that $[x] \subseteq D$ for all $x \in X$. We have, for example, $\lfloor\{\mathrm{QE} 2, \mathrm{PL} 2\}\rfloor=[\overline{\mathrm{QE}} 2]$ and $\lceil\{\mathrm{QE} 2, \mathrm{PL} 2\}\rceil=[\mathrm{PL} 2]$; and $\lfloor\{\mathrm{QE} 1, \mathrm{PL} 2\}\rfloor=\emptyset$ and $\lceil\{\mathrm{QE} 1, \mathrm{PL} 2\}\rceil=R$. Note that if there exist $x, y \in X$ such that $[x] \cap[y]=\emptyset$ then $\lfloor X\rfloor=\emptyset$.

Table 3 lists four different sets of conditions that must be satisfied for hierarchy operations to be successful. We will use these sets in the remainder of this section to prove the existence of 0SP, 1SP, 2SP and 3SP administrative models: $\mathcal{C}_{r h a}$ is the set of conditions used by the RHA family of models, and is reproduced from Table 2 for convenience; $\mathcal{C}_{i}$ gives rise to an $i \mathrm{SP}$ model, $i=0,2,3$. We also prove that $\mathcal{C}_{0}$ is sufficient to define a 1SP model.

Each column in the table specifies a set of conditions for each hierarchy operation. The conditions become increasingly restrictive from left to right. Each set of conditions is derived in part from the previous set. Note the following features of the table:

- A new condition has been introduced in order to make deleteEdge 0SP;

- New conditions are required to define 2SP operations when those operations may add edges to the hierarchy. Informally, the new conditions require that new edges are directed from children within a larger administrative domain to parents in a smaller administrative domain;

- New conditions are required to define 3SP operations. Informally, these conditions require that the most local administrator performs the operation to preserve autonomy.

Henceforth we will write $o_{x}$ to denote that we are considering hierarchy operation $o$ using conditions $\mathcal{C}_{x}$ from Table 3 . The operation addRole $2(a, r, C, P)$, for example, only succeeds if $C \subseteq \widehat{\sigma}(a), P \subseteq \sigma(a)$ and $\lceil P\rceil \subseteq\lfloor C\rfloor$.

REMARK 8. It is worth noting that each of the conditions in Table 3 can be easily checked using the domain tree. To check that $[c]=\sigma(a)$, for example, it is simply a matter of confirming that $a$ is the immediate parent of $c$ in the tree.

TheOrem 9. $\mathcal{C}_{0}$ is OSP.

\footnotetext{
${ }^{5}$ This domain is simply the (unique) immediate parent of $r$ in the domain tree. ARBAC97 defines the concept of an immediate authority range, which is analogous to this type of administrative domain.
} 


\begin{tabular}{|c|c|c|c|c|}
\hline Operation & $\mathcal{C}_{\text {rha }}$ & $\mathcal{C}_{0}$ & $\mathcal{C}_{2}$ & $\mathcal{C}_{3}$ \\
\hline addRole $(a, r, C, P)$ & $\begin{array}{l}C \subseteq \widehat{\sigma}(a) \\
P \subseteq \sigma(a)\end{array}$ & $\begin{array}{l}C \subseteq \widehat{\sigma}(a) \\
P \subseteq \sigma(a)\end{array}$ & $\begin{array}{l}C \subseteq \widehat{\sigma}(a) \\
P \subseteq \sigma(a) \\
\lceil P\rceil \subseteq\lfloor C\rfloor\end{array}$ & $\begin{array}{l}C \subseteq \widehat{\sigma}(a) \\
P \subseteq \sigma(a) \\
\lfloor C\rfloor=\lceil C\rceil=\sigma(a)\end{array}$ \\
\hline deleteRole $(a, r)$ & $r \in \widehat{\sigma}(a)$ & $r \in \widehat{\sigma}(a)$ & $r \in \widehat{\sigma}(a)$ & $\begin{array}{l}r \in \widehat{\sigma}(a) \\
{[r]=\sigma(a)}\end{array}$ \\
\hline $\operatorname{addEdge}(a, c, p)$ & $c, p \in \sigma(a)$ & $c, p \in \sigma(a)$ & $\begin{array}{l}l c, p \in \sigma(a) \\
{[p] \subseteq[c]}\end{array}$ & $\begin{array}{l}c, p \in \sigma(a) \\
{[c]=\sigma(a)}\end{array}$ \\
\hline deleteEdge $(a, c, p)$ & $c, p \in \sigma(a)$ & $c, p \in \widehat{\sigma}(a)$ & $\begin{array}{l}c, p \in \widehat{\sigma}(a) \\
\lceil\nabla p\rceil \subseteq[c]\end{array}$ & $\begin{array}{l}c, p \in \widehat{\sigma}(a) \\
{[c]=\sigma(a)}\end{array}$ \\
\hline
\end{tabular}

Table 3: Scope preserving conditions

Theorem 10. $\mathcal{C}_{0}$ is $1 \mathrm{SP}$.

TheOREM 11. $\mathcal{C}_{2}$ is 2SP.

Corollary $12 . \mathcal{C}_{3}$ is $2 \mathrm{SP}$.

TheOrem 13. $\mathcal{C}_{3}$ is 3SP.

Space constraints do not permit the inclusion of proofs of these results. Theorem 9 is established by first proving that RHA operations preserve both $\downarrow a$ and $\uparrow a$, except for deleteEdge $_{r h a}$. The proof of this preparatory result is constructive and yields the appropriate condition for deleteEdge to be 0SP. (Notice that columns two and three of Table 3 only differ in the entry for the delete edge operation.) Theorem 10 is proved by extending the proof method used for Theorem 9. Theorem 11 is proved using the fact that domains are either nested or disjoint and that $\mathcal{C}_{2}$ only permits the addition of edges to the hierarchy if they are directed into interior domains, thereby preserving the set of senior roles of the child role. Corollary 12 is established by proving that if operation $o p_{3}$ succeeds then so does $o p_{2}$ and then using Theorem 11, and again makes use of the fact that domains are nested. Theorem 13 follows from the definition of $\mathcal{C}_{3}$ and a simple proof by contradiction. Full proofs are available in our technical report [5].

\section{RBAT: A TEMPLATE FOR ROLE- BASED ADMINISTRATIVE MODELS}

We have introduced the idea of an administrative domain and a number of criteria that can be used to control the way in which administrative domains are affected by hierarchy operations. In this section we briefly describe RBAT (role-based administration template), which provides a design pattern for role-based administration models. We will show how particular instances of the framework are related to $\mathrm{RHA}_{4}$ and ARBAC97.

\subsection{Components of RBAT}

RBAT defines the following components:

- A non-empty set of administrative units $\mathcal{U}$, each of which contains a unique administrator role. Moreover, for all $U, U^{\prime} \in \mathcal{U}$, one of the following conditions must hold: (i) $U \subseteq U^{\prime}$ (ii) $U \supseteq U^{\prime}$ (iii) $U \cap U^{\prime}=\emptyset$;

- A set of hierarchy operations $\mathcal{O}$;

- A set of conditions $\mathcal{C}$, each of which determines the success of a particular operation;
- A set of administrative roles $R_{A}$, which may be empty;

- A relation can-administer $\subseteq R_{A} \times R$, which associates an administrative role with the administrator of an administrative unit. If $R_{A}=\emptyset$, can-administer $\subseteq$ $R \times R$.

\subsection{The can-administer relation}

Instead of using roles in the hierarchy, we may define a distinct set of administrative roles and assign them to administrative domains within the role hierarchy. This is similar to the approach taken in ARBAC97 and is a simplification of the admin-auth relation in the RHA family of models.

Since an administrative domain is uniquely determined by its administrator, we can introduce a relation can-administer $\subseteq R_{A} \times R$, where $R_{A}$ is the set of administrative roles. The meaning of $(a, r) \in$ can-administer is that $a$ has administrative control of $\sigma(r)$, the administrative unit defined by its administrator $r$. (For simplicity, we continue to use $\sigma(r)$ to denote the administrative unit defined by an administrator $r$; we will also write $\sigma(a)$ to denote the set of administrative units controlled by an administrative role a.) Hence (PS01, PL1) $\in$ can-administer, for example, could be used to specify that PSO1 has been granted control over the administrative unit $\sigma(\mathrm{PL} 1)=\{$ ENG1, PE1, QE1, PL1 $\}$.

Table 4 shows the conditions for success of hierarchy operations within this general framework. In simple terms, an operation performed by an administrative role $a$ succeeds if all the arguments of the operation belong to a single administrative unit (with administrator $x$ ) that is controlled by $a$ and $x$ is permitted to perform the desired operation within that administrative unit. The model can be chosen to be 0SP, 1SP, 2SP or 3SP, simply by selecting the appropriate criteria for the operation to succeed when performed by $x$. If (PSO1, PL1) $\in$ can-administer, for example, then deleteEdge ${ }_{r h a}(\mathrm{PSO} 1, \mathrm{PE} 1, \mathrm{PL} 1)$ succeeds, but deleteEdge $_{0}$ (PSO1, PE1, PL1) fails.

\subsection{The RHA model $_{4}$}

It is natural to expect that RBAT has some similarity with the RHA family of models, since administrative units are a generalization of administrative domains (which are defined by administrative scope). Note that $\mathrm{RHA}_{1}$ is a special case of RBAT in which $\mathcal{U}$ is the set of administrative domains, $R_{A}=\emptyset$, can-administer $=\{(r, r): r \in R\}$ and $\mathcal{C}=\mathcal{C}_{r h a}$.

The can-administer relation is identical in structure to the relation admin-auth $\subseteq R_{A} \times R$ defined in $\mathrm{RHA}_{4}$, the most complex model of the RHA family. In RBAT, we define the administrative scope of an administrative role $a$ to be the 


\begin{tabular}{|l|l|l|}
\hline Operation & Conditions & addRole $(x, r, C, P)$ succeeds \\
\hline addRole $(a, r, C, P)$ & & deleteRole $(x, r)$ succeeds \\
deleteRole $(a, r)$ & $\exists x \in R,(a, x) \in$ can-administer & addEdge $(x, c, p)$ succeeds \\
addEdge $(a, c, p)$ & deleteEdge $(x, c, p)$ succeeds \\
deleteEdge $(a, c, p)$ & & deleter
\end{tabular}

Table 4: Success of hierarchy operations in RBAT

union of the administrative units it controls and insist that for any command to succeed, all arguments must belong to a single one of those units. However, in $\mathrm{RHA}_{4}$, the administrative scope of $a$ was defined in terms of the roles controlled by $a$ (that is, $\{r \in R:(a, r) \in$ admin-auth $\}$ ). An example should make the difference clearer: the RHA model would permit (PS01, PE1), (PS01, QE1) $\in$ admin-auth, meaning that $\sigma(\mathrm{PSO} 1)=\{$ ENG1, PE1, QE1 $\}$, whereas these pairs are not permitted in the can-administer relation because PE1 and QE1 are not administrators in $R$. Moreover, although we permit (PS01, PL1), (PS01, PL2) $\in$ can-administer, for example, we do not permit the operation addEdge ${ }_{2}$ (PSO1, ENG1, QE2). Strictly speaking, then, $\mathrm{RHA}_{4}$ is not an instance of RBAT, although an OSP model that uses administrative domains as administrative units is a close approximation to $\mathrm{RHA}_{4}$.

\section{CONNECTIONS WITH ARBAC97}

What is more surprising is that the ARBAC97 model can be expressed in terms of the framework described in the last section. ARBAC97 defines the relation can-modify $\subseteq R_{A} \times \mathcal{E}$, where $\mathcal{E}$ is the set of encapsulated ranges in $R$ (see Definition 14 below). Roughly speaking, the administrative role $a \in R_{A}$ can perform a hierarchy operation provided the arguments are contained in some encapsulated range $E$ and $(a, E) \in$ can-modify. In addition, no hierarchy operation may violate the encapsulation of the ranges contained in the can-modify relation: this is clearly a kind of preservation property.

In this section we identify a strong link between encapsulated ranges and administrative domains and provide a new formulation of ARBAC97. We also identify a couple of weaknesses in the original formulation, which become apparent when the ARBAC97 model is interpreted within our framework.

The following definition is due to Sandhu et al [15, Definition 16], although it has been slightly modified as a result of an observation made by Crampton and Loizou [6, Remark $7.3]$.

Definition 14. A range $[x, y]$ is encapsulated if for all $z \in(x, y)$ and all $w \notin(x, y)$ :

$$
\begin{aligned}
& w>z \text { iff } w \geqslant y \\
& w<z \text { iff } w \leqslant x .
\end{aligned}
$$

Lemma 15. For any encapsulated range $[x, y]$, $\lceil[x, y]\rceil=\sigma(y)$.

Proof. Let $[x, y]$ be an encapsulated range and let $z \in[x, y]$. We will show that $\uparrow z \subseteq \uparrow y$. Let $w \in \uparrow z$. There are two possibilities: (i) if $w \leqslant y$ then $w \in \uparrow y$; (ii) if $w \nless x$ then since $z \leqslant w$ and $[x, y]$ is encapsulated we have $y \leqslant w$ and $w \in \uparrow y$. Hence, for all $z \in[x, y], z \in \sigma(y)$ and $[z] \subseteq \sigma(y)$. The result follows by observing that any smaller administrative domain does not contain $y$.
COROLlaRY 16. Encapsulated ranges are either nested or disjoint.

Proof. The result follows immediately from Lemma 2 and Lemma 15.

The converse of Lemma 15 is not true because an administrative domain is not necessarily a range. However, we have the following definition and result.

DEFINITION 17. An administrative range is a range $[b, t] \in R$ such that for all $x \in[b, t], \uparrow x \subseteq \uparrow t$ and $\downarrow x \subseteq \uparrow b .{ }^{6}$

Note that the definition of administrative range is the symmetric analogue of the definition of administrative scope. We will use this fact later when deriving conditions for an operation to preserve encapsulated ranges.

Proposition 18. The range $[b, t]$ is encapsulated iff $[b, t]$ is an administrative range.

Proof. The result follows from the definition of administrative range and the proof method of Lemma 15.

Note that an encapsulated range does not include the end points that define it. In other words, an encapsulated range is analogous to strict administrative scope (which omits the top element in an administrative domain). To avoid the introduction of any further notation, we will write $\widehat{\sigma}(a)$ to denote the encapsulated range with top element $a$.

In ARBAC97, the ranges that appear in the can-modify relation are called authority ranges. They are defined by the system administrator and are required to be encapsulated ranges. Moreover, it is required that each pair of authority ranges be either nested or disjoint. Corollary 16 shows that this requirement is redundant as encapsulated ranges are either nested or disjoint by definition.

The success of many operations in ARBAC97 depends on the notion of an immediate authority range. Since authority ranges are nested or disjoint by definition, there exists a smallest authority range to which any given role belongs. The immediate authority range of a role $r$ is analogous to $[r]$.

We now place ARBAC97 in the context of the framework developed in this paper. In ARBAC97, every hierarchy operation must preserve the encapsulation of all authority ranges. The designers of ARBAC97 give no rules or method for determining whether a hierarchy operation satisfies this condition. It should come as no surprise by now that we are able to express ARBAC97 using the approach described in the previous section and that we can explicitly state sufficient conditions for an operation to preserve encapsulated ranges. Specifically, $\mathcal{U}$ is defined to be the set of encapsulated ranges, $\mathcal{C}$ is the analogue of $\mathcal{C}_{2}$ for encapsulated ranges, and can-modify is replaced by can-administer.

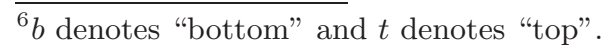


In Table 5 we summarize the conditions that must be satisfied for a hierarchy operation to succeed in the ARBAC97 model. We write $x \in \sigma(a)$ as an abbreviation for "there exists $r \in R$ such that $x \in \sigma(r)$ and $(a, r) \in$ can-administer". Expressions such as $X \subseteq \sigma(a)$ and $x \in \widehat{\sigma}(a)$ have analogous interpretations.

The second column restates the conditions given by Sandhu in the original formulation of the model. Notice the use of $\widehat{\sigma}(a)$ in the second column, corresponding to the fact that the basic unit of administration in ARBAC97 is the encapsulated range, which does not include the end points of the range. We have simplified some of the conditions for the addRole and addEdge operations, which were permitted in the original formulation of ARBAC97 provided one of three conditions was satisfied, one of which was that $[c]=[p]$. In fact, each of these conditions turns out to be equivalent. By symmetry, and using $\mathcal{C}_{2}$ from Table 3 , all encapsulated ranges are preserved if $[c] \subseteq[p]$ and $[p] \subseteq[c]$. Therefore, addRole $(a, r,\{c\},\{p\})$ succeeds if $c, p \in \widehat{\sigma}(a)$ and $[c]=[p]$.

The third column suggests some slight modifications to these conditions that should yield an improved version of ARBAC97. Specifically, we add a condition to the deleteEdge operation that guarantees that all such operations preserve encapsulated ranges. Sandhu et al do not comment on the fact that deleting an edge can destroy an encapsulated range and make no effort to prevent this happening. In contrast, we introduced a new requirement into $\mathcal{C}_{2}$ for the deleteEdge operation in order to preserve administrative domains. Correspondingly, ARBAC97 should include the following condition for the deleteEdge operation in order to preserve all encapsulated ranges: $[p]=[c]$, since we require that $[\nabla p] \subseteq[c]$ and (by symmetry) $[\Delta c] \subseteq[p]$ in order to preserve encapsulated ranges. We also make the definition of addRole more general, in line with the addRole operation used elsewhere in this paper. The ARBAC97 model requires that a new role have a single child and parent role. There is no theoretical reason for this restriction. Hence we suggest that we use the operation addRole $(a, r, C, P)$, and that it succeeds if $C \subseteq \widehat{\sigma}(a), P \subseteq \sigma(a)$ and $\lfloor C\rfloor=\lceil C\rceil=\lfloor P\rfloor=$ $\lceil P\rceil$. (This latter condition simply says that there exists $b$ such that for all $c \in C$ and all $p \in P,[c]=[p]=\sigma(b)$.)

\section{CONCLUSION}

We have provided a characterization of role-based administrative models based on the extent to which the hierarchy operations permitted by the model preserve administrative domains. This characterization enables us to provide a concise description of ARBAC97 and to identify and correct a number of flaws in the original specification.

The success or otherwise of hierarchy operations is determined by the administrative scope of the role performing the operation. The administrative scope of a role can be determined directly and efficiently from the domain tree. Hence it ought to be possible to produce an implementation of ARBAC97 and a number of other models described in this paper for evaluation purposes. Until now, it was not obvious that such an implementation of ARBAC97 existed, since there was no obvious way of testing the requirement that the encapsulation of all authority ranges be preserved.

RBAT mandates the specification of a set of administrative units, which must be either pairwise nested or disjoint. The obvious choices for such a set are administrative domains and encapsulated ranges (giving rise to RHA-style and ARBAC97-style models respectively). However, these are not the only choices and we may also consider the administration of disconnected hierarchies (such as those defined in ERBAC96 [14] and TRBAC [3]). In this instance, it will be necessary for systems administrators to define the administrative domains (that is, without reference to administrative scope or encapsulated ranges) and then use an appropriate set of conditions to determine the success of hierarchy operations and preserve the integrity of those administrative domains. Of course this will require some modification to the conditions $\mathcal{C}_{0}, \mathcal{C}_{2}$ and $\mathcal{C}_{3}$, because in these conditions the notion of preserving administrative domains could be neatly captured using the administrative scope of a role. Instead, the conditions will have to be specified in terms of preserving $\downarrow r$, where $r$ is the administrator of the domain, and $\uparrow x$, where $x$ belongs to the domain of which $r$ is the administrator. This does not appear to present insuperable difficulties. To date no administrative model exists for either ERBAC96 or TRBAC.

We can also build real ARBAC97 systems. This was not obvious previously, since there existed a "chicken and egg" situation, in which the can-modify relation was defined in terms of the hierarchy, but the can-modify relation controlled changes to the hierarchy [6, Section 8.3$]$. We know that we can build RHA systems if we assume the existence of a system administrator role that initially controls an empty domain [6], which therefore suggests that we can also build ARBAC97 systems.

Moreover, we can define more "relaxed" ARBAC97-style models. The original version of the model is essentially a 2SP model, since it requires that all encapsulated ranges be preserved. We now have a framework that enables us to develop a set of less restrictive models based on authority ranges, but with weaker preservation properties such as OSP.

Finally, we note that this work may have a considerable impact on the study of the safety problem in role-based systems $[4,11,12]$. The safety problem considers the propagation of access rights due to changes to access control data structures and hence every administrative model gives rise to an instance of the safety problem. The fact that we can order administrative models according to the extent to which they preserve domains may enable us to identify classes of rolebased administrative systems which have a decidable safety problem. We anticipate that domain preservation will be a feature of models for which the safety problem is decidable in polynomial time.

We believe that this work will be of benefit to application and systems developers, who wish to understand the mechanisms of role-based administration better and to know what properties will be preserved by the administrative model they choose to implement. We also believe this work lays a valuable theoretical foundation for the further development of role-based administrative models and investigation of the safety problem in role-based systems.

Future work will include the construction of administrative models for ERBAC96 and TRBAC as suggested above. We will also extend RBAT to include all administrative operations (as defined in Section 2) and the administration of the can-administer relation. A further interesting possibility is to introduce administrative permissions. This leads to a two-phase checking process for administrative operations, similar to that in the Bell-LaPadula model: the operation should be both explicitly permitted by the assignment of appropriate administrative permissions and should satisfy the conditions for the operation to proceed. In this context, 


\begin{tabular}{|l|l|l|}
\hline Operation & Sandhu et al & Crampton \\
\hline \multirow{5}{*}{ addRole $(a, r, C, P)$} & $C=\{c\}$ & $C \subseteq \widehat{\sigma}(a)$ \\
& $P=\{p\}$ & $P \subseteq \sigma(a)$ \\
& $c, p \in \widehat{\sigma}(a)$ & $\lfloor C\rfloor=\lceil C\rceil=\lfloor P\rfloor=\lceil P\rceil$ \\
\hline deleteRole $(a, r)$ & $r \in \widehat{\sigma}(a)$ & $r \in \widehat{\sigma}(a)$ \\
\hline \multirow{2}{*}{ addEdge $(a, c, p)$} & $c, p \in \widehat{\sigma}(a)$ & $c, p \in \sigma(a)$ \\
& {$[c]=[p]$} & {$[c]=[p]$} \\
\hline \multirow{2}{*}{ deleteEdge $(a, c, p)$} & $c, p \in \widehat{\sigma}(a)$ & $c, p \in \widehat{\sigma}(a)$ \\
& & {$[c]=[p]$} \\
\hline
\end{tabular}

Table 5: Hierarchy operations in ARBAC97

the conditions form the mandatory element of the access control checking process and the permissions form the discretionary element. The introduction of administrative permissions also suggests the possibility of administrative separation of duty. We could, for example, insist that for a given administrative domain, a human resources role is responsible for the administration of the user-role assignment relation, whereas some managerial or systems administrator role is responsible for the administration of the permission-role assignment relation. Finally, we hope to investigate the notion of role "visibility", which we regard as being analogous to the scope of a variable in block structured programming languages. Some roles and edges within a domain might only be visible to roles within that domain, whereas other might have global visibility. This concept may help to address the fact that it is not always appropriate for all permissions to be available to all more senior roles. Certainly, there is no shortage of directions in which this work can be developed.

Acknowledgements. The author would like to thank the anonymous reviewers for their constructive comments and suggestions.

\section{REFERENCES}

[1] Abrams, M., LaPadula, L., and Olson, I. M. Building generalized access control on UNIX. In Proceedings of Second USENIX UNIX Security Workshop (1990), pp. 65-70.

[2] American National Standards Institute. ANSI INCITS 359-2004 for Role Based Access Control, 2004.

[3] Bertino, E., Bonatti, P., and Ferrari, E. TRBAC: A temporal role-based access control model. ACM Transactions on Information and System Security 4, 3 (2001), 191-223.

[4] Crampton, J. Authorization and antichains. PhD thesis, Birkbeck, University of London, London, England, 2002. Available from http://www.isg.rhul.ac.uk/ jason.

[5] Crampton, J. Understanding and developing role-based administrative models. Tech. Rep. RHUL-MA-2005-6, Royal Holloway, University of London, 2005.
[6] Crampton, J., And Loizou, G. Administrative scope: A foundation for role-based administrative models. ACM Transactions on Information and System Security 6, 2 (2003), 201-231.

[7] Davey, B., AND Priestley, H. Introduction to Lattices and Order. Cambridge University Press, Cambridge, United Kingdom, 1990.

[8] Ferraiolo, D., and Kuhn, D. Role-based access control. In Proceedings of the 15th NIST-NSA National Computer Security Conference (1992), pp. $554-563$.

[9] Ferraiolo, D., Sandhu, R., Gavrila, S., Kuhn, D., And Chandramouli, R. Proposed NIST standard for role-based access control. ACM Transactions on Information and System Security 4, 3 (2001), 224-274.

[10] Harrison, M., Ruzzo, W., And Ullman, J. Protection in operating systems. Communications of the ACM 19, 8 (1976), 461-471.

[11] Li, N., And Tripunitara, M. Security analysis in role-based access control. In Proceedings of the Ninth ACM Symposium on Access Control Models and Technologies (2004), pp. 126-135.

[12] Munawer, Q., And Sandhu, R. Simulation of the augmented typed access matrix model (ATAM) using roles. In Proceedings INFOSECU99 International Conference on Information Security (1999).

[13] Nyanchama, M., And Osborn, S. The role graph model and conflict of interest. ACM Transactions on Information and System Security 2, 1 (1999), 3-33.

[14] SAndhu, R. Role activation hierarchies. In Proceedings of Third ACM Workshop on Role-Based Access Control (1998), pp. 33-40.

[15] Sandhu, R., Bhamidipati, V., and Munawer, Q. The ARBAC97 model for role-based administration of roles. ACM Transactions on Information and System Security 1, 2 (1999), 105-135.

[16] Sandhu, R., Coyne, E., Feinstein, H., And Youman, C. Role-based access control models. IEEE Computer 29, 2 (1996), 38-47. 